\title{
Lesion Characteristics of Chronic Dysphagia in Patients With Supratentorial Stroke
}

\author{
Sol Jang, MD, Hea Eun Yang, MD, Hee Seung Yang, MD, Dae Hyun Kim, MD
}

Department of Physical Medicine and Rehabilitation, Veterans Health Service Medical Center, Seoul, Korea

\begin{abstract}
Objective To analyze the relationship between brain lesion location and type of chronic dysphagia in patients with supratentorial stroke.

Methods Data from 82 chronic stroke patients who underwent videofluoroscopic swallowing studies at $>6$ months after an initial stroke event were retrospectively analyzed. Delayed oral transit time, delayed pharyngeal transit time, and the presence of aspiration were extracted. A voxel-based lesion symptom mapping (VLSM) analysis was used to correlate types of dysphagia with specific brain lesions.

Results VLSM identified several clusters of voxels that significantly correlated with type of dysphagia. Delayed oral transit time mainly correlated with lesions in the left inferior frontal lobe and precentral gyrus; delayed pharyngeal time mainly correlated with lesions in the right basal ganglia and corona radiate; and aspiration was mainly correlated with lesions in the putamen.

Conclusion Understanding the association between lesion location and dysphagia in chronic stroke patients is an important first step towards predicting permanent dysphagia after stroke. Improved understanding of the neural correlates of dysphagia will inform the utility of interventions for its treatment and prevention after stroke.
\end{abstract}

Keywords Dysphagia, Video fluoroscopy, Stroke, Neuroimaging

\section{INTRODUCTION}

Dysphagia is a common complication of stroke that occurs in $33 \%-73 \%$ of stroke patients [1]. It can cause serious complications such as aspiration pneumonia, dehydration, malnutrition, and death $[2,3]$. Whereas dysphagia typically resolves within 2 weeks in most poststroke patients, it persists in about $10 \%$ of individuals [4].
The incidence of dysphagia is significantly associated with stroke lesions of the brain stem and/or the bilateral hemispheres [5]. Previous studies have identified lesion patterns that can predict dysphagia after acute stroke $[4,6,7]$. However, these studies were limited to the acute period after stroke. Moreover, lesions outside of the brainstem have not been investigated in the context of chronic dysphagia. Thus, the aim of this study was to

Received April 28, 2016; Accepted June 14, 2016

Corresponding author: Dae Hyun Kim

Department of Physical Medicine and Rehabilitation, Veterans Health Service Medical, 53 Jinhwangdo-ro 61-gil, Gangdong-gu, Seoul 05368, Korea. Tel: +82-2-2225-4177, Fax: +82-2-2225-4177, E-mail: hohoho7490@gmail.com

ORCID: Sol Jang (http://orcid.org/0000-0002-8534-9599); Hea Eun Yang (http://orcid.org/0000-0002-4449-7288); Hee Seung Yang (http://orcid. org/0000-0003-2492-7065); Dae Hyun Kim (http://orcid.org/0000-0002-5065-4286).

(c) This is an open-access article distributed under the terms of the Creative Commons Attribution Non-Commercial License (http://creativecommons.org/ licenses/by-nc/4.0) which permits unrestricted noncommercial use, distribution, and reproduction in any medium, provided the original work is properly cited. Copyright (C) 2017 by Korean Academy of Rehabilitation Medicine 
evaluate potential correlations between supratentorial lesion locations and types of chronic dysphagia using voxel-based lesion symptom mapping (VLSM) and videofluoroscopic swallowing studies (VFSS).

\section{MATERIALS AND METHODS}

\section{Subjects}

We screened 587 consecutive patients who underwent VFSS at the Veterans Health Service Medical Center between March 2014 and February 2015. A total of 82 chronic patients (mean age, 73.9 years; 75 males and 7 females) met the inclusion criteria of this retrospective study. The inclusion criteria were (1) confirmation of a first stroke event with magnetic resonance imaging (MRI) or computed tomography, (2) supratentorial stroke, (3) an interval of least 6 months since stroke, and (4) MRI and VFSS conducted with a maximum interval of one week. Exclusion criteria were (1) a previous history of stroke and (2) any coexisting neurological disease that could influence swallowing function. Among the 82 supratentorial stroke patients recruited to the study, 68 patients had ischemic stroke and 14 had hemorrhagic stroke. Eleven strokes occurred in the right hemisphere, 26 occurred in the left hemisphere, and 45 occurred in the bilateral hemispheres. Fig. 1 summarized the lesion data. The demographic and clinical characteristics of included patients were shown in Table 1. This study was approved by our Institutional Research Ethics Committee for Human Subjects.

\section{Dysphagia assessment}

Patients were seated in an upright position on a wheelchair and X-ray images of the head, neck, and upper chest were acquired in real time after dosage with each fluid material, except for the $10 \mathrm{~mL}$ water test. Each dysphagia bolus was followed radiographically through the mouth, throat, and into the esophagus. Depending on the facility, a simple screening sequence of subsequent bolus movement down the esophagus was also recorded. Both lateral and anterior-posterior views were acquired. Five types of barium-impregnated boluses of different consistency $(2,5$, or $10 \mathrm{~mL}$ water, 2 or $5 \mathrm{~mL}$ plain-yogurt, $5 \mathrm{~mL}$ boiled-rice, or banana and cookie in 33\% liquid barium sulfate) were used in swallowing tests. Dysphagia types were diagnosed based on a review of VFSS records. Delayed oral transit time (OTT), delayed pharyngeal transit time (PTT), and aspiration were noted for all patients. OTT was defined as the time from initial backward movement of the bolus until the bolus reached the lower edge of the mandible. PTT was defined as the time from the arrival of the bolus at the lower edge of the mandible until the bolus tail passed through the cricopharyngeal region [8]. Delayed OTT and PTT were defined by transit durations $>1.5$ seconds. Aspiration was defined as the presence of bolus material beyond the level of the true

Table 1. Clinical characteristics of patients

\begin{tabular}{|lc|}
\hline \multicolumn{1}{|c|}{ Characteristic } & Value \\
\hline Sex & \\
\hline Male & $75(91.5)$ \\
\hline Female & $7(8.5)$ \\
\hline Age (yr) & $73.9 \pm 8.01$ \\
\hline Lesion side & \\
\hline Right & $26(31.7)$ \\
\hline Left & $11(13.4)$ \\
\hline Bilateral & $45(54.9)$ \\
\hline Type of stroke & $68(82.9)$ \\
\hline Ischemic & $14(17.1)$ \\
\hline Hemorrhagic & $35.23 \pm 45.89$ \\
\hline Duration of disease $(\mathrm{mo})$ & \\
\hline
\end{tabular}

Values are presented as number of patients (\%) or mean \pm standard deviation.

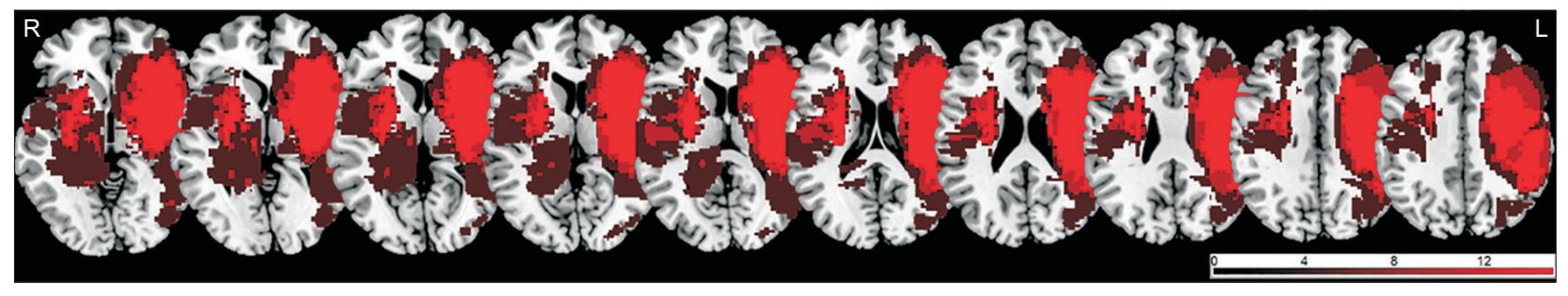

Fig. 1. Lesion overlapping maps. 
vocal cord [8]. VFSS results, including OTT, PTT, and penetration or aspiration, were performed and interpreted by two well-trained rehabilitation medicine physicians.

\section{Magnetic resonance image acquisition}

All images were acquired using a 3-T clinical wholebody magnetic resonance scanner (Siemens, Erlangen, Germany) with a 20-channel head coil. High-resolution 3D T1-weighted images were obtained. The 3D T1weighted imaging parameters were as follows: repetition time/echo time, 1900/2.57 ms; matrix, $256 \times 256$; field of view, $230 \mathrm{~mm} \times 230 \mathrm{~mm}$; flip angle, 9; and slice thickness, $1 \mathrm{~mm}$.

\section{Voxel-based lesion symptom mapping and statistical} analysis

Lesions were visually identified by altered $\mathrm{T} 1$ signal intensity. The identification procedure was as follows: first, borders for each lesion were drawn on high-resolution T1-weighted images in the native space using MRIcro software (http://www.mricro.com). Second, T1-weighted images and native space lesion images were non-linearly transformed to the standardized Montreal Neurologi- cal Institute (MNI) space using SPM8 software (http:// www.fil.ion.ucl.ac.uk/spm). Associations between lesion locations and types of dysphagia were analyzed with the VLSM method implemented in the nonparametric mapping tool of MRIcron software [9]. For statistical purposes, we considered delayed OTT, delayed PTT, and aspiration as binary variables (absent/present) based on a binary images/binary behavior design $[9,10]$. The level of significance was set at an uncorrected $\mathrm{p}<0.005[10,11]$. Lesions were traced by an experienced neurorehabilitation physician who was blinded to all clinical data.

\section{RESULTS}

\section{Dysphagia assessment and lesion associations}

Twenty-two patients had delayed OTT, 8 had delayed PTT, and 30 presented with aspiration. Left hemispheric lesions of the inferior frontal lobe and precentral gyrus were significantly correlated with delayed OTT (uncorrected $\mathrm{p}<0.005$ ) (Fig. 2A). Right hemispheric lesions of the basal ganglia and corona radiata were significantly correlated with delayed PTT (uncorrected p<0.005) (Fig. 2B). Finally, aspiration was significantly correlated with
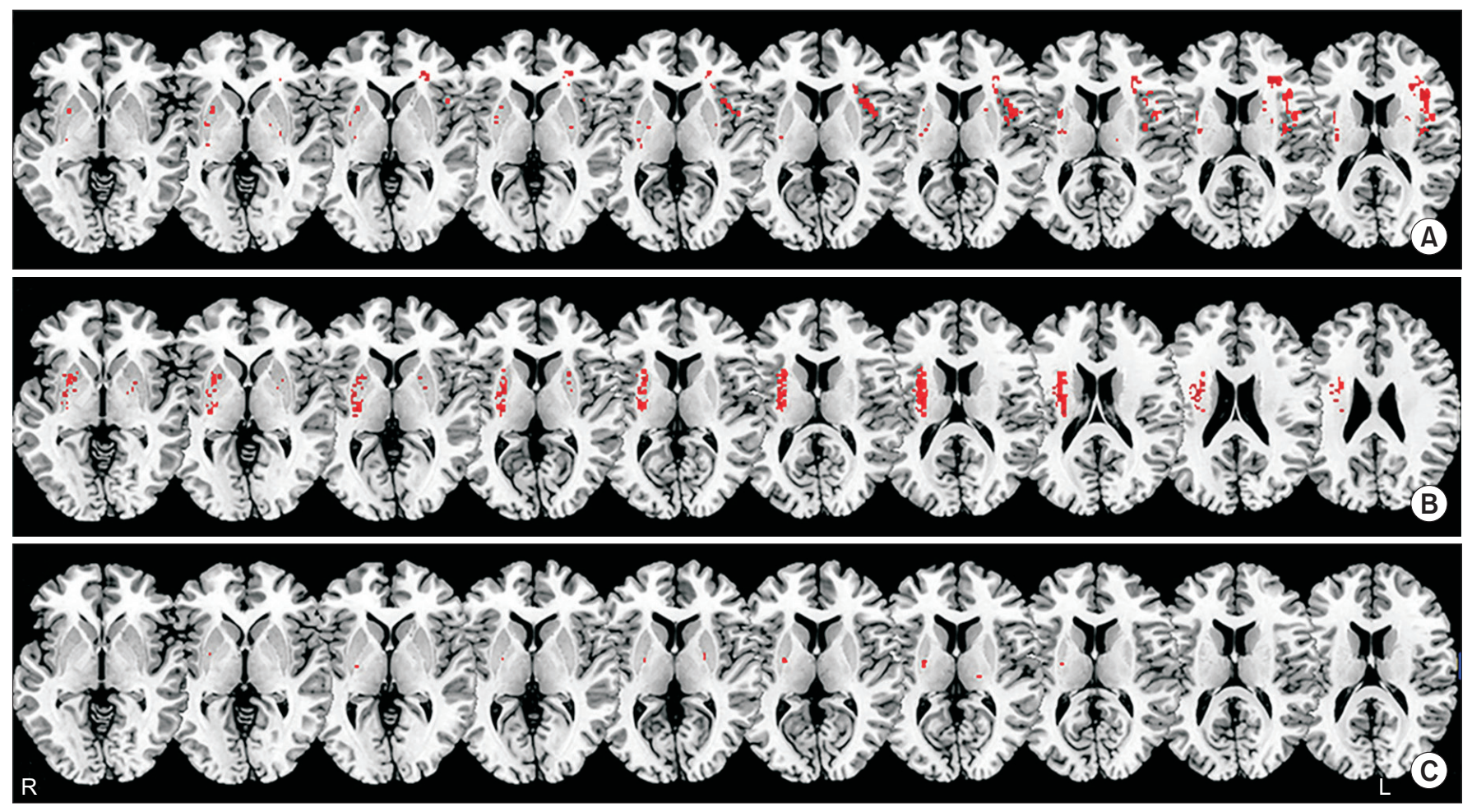

Fig. 2. Voxel-based lesion symptom mapping results showing voxels in patients with dysphagia. (A) Correlation with delayed oral transit time. (B) Correlation with delayed pharyngeal transit time. (C) Correlation with the presence of aspiration. 
right hemispheric lesions of the putamen (uncorrected $\mathrm{p}<0.005$ ) (Fig. 2C).

\section{DISCUSSION}

The present study indicated significant correlations between lesion location and type of dysphagia in stroke patients with permanent dysphagia. Oral phase problems were mainly correlated with lesions of the left inferior frontal lobe and left precentral gyrus, whereas pharyngeal phase impairments were correlated with lesions of right basal ganglia, corona radiata, and aspiration was mainly correlated with lesions of the right putamen.

In cases of stroke, dysphagia is traditionally associated with brainstem lesions or bilateral cortical damage $[12,13]$. However, recent studies have identified dysphagia in patients with unilateral hemispheric lesions $[4,5,7,12,14]$. At least $40 \%$ of patients with unilateral hemispheric stroke reportedly have swallowing difficulties [15-17]. Because swallowing requires complex neuromuscular activity, unilateral hemispheric damage can lead to dysphagia after stroke.

Swallowing occurs in voluntary (oral) and involuntary (pharyngeal) phases, hence, damage to voluntary and involuntary components of neurological function can affect dysphagia after a stroke event [18]. Further, swallowing utilizes bi-hemispheric components of neurological function $[12,19]$. In our results, the left hemisphere was more associated with the voluntary oral phase of swallowing, whereas the right hemisphere was more associated with the reflexive (involuntary) pharyngeal phase and aspiration as a complication. Our results corroborate those of previous studies analyzing dysphagia by location of cortical lesions [4,5]. Daniels et al. [7] concluded that the left hemisphere plays a major role in the oral phase of swallowing, while the right hemisphere dominantly coordinates the pharyngeal stage. Watanabe et al. [20] also found that activation of the insula was strongly lateralized to the left hemisphere in the initiation stages of swallowing using magnetoencephalography. Other studies have suggested similar right-left differences, with prolonged pre-pharyngeal response times associated with left-hemispheric stroke and more severe pharyngeal dysfunction associated with right-hemispheric stroke [21-23]. These results support lateralization of specific swallowing functions observed in our study.
With regards to oral phase swallowing dysfunction, post-stroke lesions of the corticospinal and corticobulbar tracts interrupt voluntary control of mastication and bolus transport. Cola et al. [24] showed that disruption of anatomical connectivity tracts was associated with dysphagia in $60 \%$ of acute stroke patients. Wan et al. [25] also showed that damage to swallowing-related cortical areas resulted in prolonged OTT. These data support our current finding that injury to the inferior frontal cortex and precentral gyrus correlate with voluntary oral phase dysfunction.

Pharyngeal dysfunction involves relatively large brain areas including the corona radiate. Heilman and Van Den Abell [26] reported that neural pathways involved in activation of the motor system are asymmetrically organized in humans; whereas, the right hemisphere plays a dominant role in activating both ipsilateral and contralateral motor neurons, the left hemisphere mainly activates ipsilateral motor neurons. Howes and Boller [27] observed reduced activation in patients with right hemispheric lesions. Thus, similar mechanisms may affect the motor neuron pools involved in swallowing and the cough response in patients with right hemispheric lesions. Studies have also confirmed an association between right hemispheric lesions and aspiration due to dysphagia [5,28], in agreement with our finding that damage to the right putamen was strongly associated with aspiration in patients with chronic dysphagia after supratentorial stroke.

The present study had several limitations. First, the study was conducted as a retrospective analysis. Second, the present study had small subgroup sample sizes for stroke and dysphagia types. Third, more than half of the patients had bilateral hemispheric stroke. Although we included only first-time stroke patients, a high percentage of individuals had previous non-symptomatic small visible lesions on brain MRI. The presence of previous damage without clinical symptoms may have influenced the dysphagia manifestation; thus, lesions of bilateral hemisphere were included for the VLSM analysis. Despite these limitations, this is the first study to show a specific correlation between lesions in the supratentorial hemisphere and chronic dysphagia using a VLSM analysis. Future studies on VFSS factors related to swallowing processing including apraxia, the Postural Assessment Scale for Stroke scores, the National Institutes of Health Score Scale ratings, residues, and esophageal phase ab- 
normalities are required to validate and extend the current findings.

In conclusion, characteristics of swallowing difficulty in supratentorial stroke patients with dysphagia varied in terms of OTT, PTT and aspiration based on brain lesion location. Our results could facilitate clinical diagnosis of chronic dysphagia and suggest that the nature of brain damage after stroke can be a useful predictor of permanent dysphagia. Moreover, the present study may help practitioners to better identify high-risk patients in the acute stage of stroke.

\section{CONFLICT OF INTEREST}

No potential conflict of interest relevant to this article was reported.

\section{REFERENCES}

1. Mann G, Hankey GJ, Cameron D. Swallowing disorders following acute stroke: prevalence and diagnostic accuracy. Cerebrovasc Dis 2000;10:380-6.

2. Martino R, Foley N, Bhogal S, Diamant N, Speechley M, Teasell R. Dysphagia after stroke: incidence, diagnosis, and pulmonary complications. Stroke 2005;36: 2756-63.

3. Han H, Shin G, Jun A, Park T, Ko D, Choi E, et al. The relation between the presence of aspiration or penetration and the clinical indicators of dysphagia in poststroke survivors. Ann Rehabil Med 2016;40:88-94.

4. Steinhagen V, Grossmann A, Benecke R, Walter U. Swallowing disturbance pattern relates to brain lesion location in acute stroke patients. Stroke 2009;40:1903-6.

5. Daniels SK, Foundas AL, Iglesia GC, Sullivan MA. Lesion site in unilateral stroke patients with dysphagia. J Stroke Cerebrovasc Dis 1996;6:30-4.

6. Galovic M, Leisi N, Muller M, Weber J, Abela E, Kagi G, et al. Lesion location predicts transient and extended risk of aspiration after supratentorial ischemic stroke. Stroke 2013;44:2760-7.

7. Daniels SK, Corey DM, Fraychinaud A, DePolo A, Foundas AL. Swallowing lateralization: the effects of modified dual-task interference. Dysphagia 2006;21: 21-7.

8. Logemann JA. Evaluation and treatment of swallowing disorders. 2nd ed. Austin: Pro-ED; 1998.
9. Rorden C, Karnath HO, Bonilha L. Improving lesionsymptom mapping. J Cogn Neurosci 2007;19:1081-8.

10. Picelli A, Tamburin S, Gajofatto F, Zanette G, Praitano $\mathrm{M}$, Saltuari L, et al. Association between severe upper limb spasticity and brain lesion location in stroke patients. Biomed Res Int 2014;2014:162754.

11. Rorden C, Bonilha L, Nichols TE. Rank-order versus mean based statistics for neuroimaging. Neuroimage 2007;35:1531-7.

12. Robbins J, Levine RL, Maser A, Rosenbek JC, Kempster GB. Swallowing after unilateral stroke of the cerebral cortex. Arch Phys Med Rehabil 1993;74:1295-300.

13. Horner J, Massey EW, Brazer SR. Aspiration in bilateral stroke patients. Neurology 1990;40:1686-8.

14. Dziewas R, Soros P, Ishii R, Chau W, Henningsen H, Ringelstein EB, et al. Neuroimaging evidence for cortical involvement in the preparation and in the act of swallowing. Neuroimage 2003;20:135-44.

15. Martin RE, Sessle BJ. The role of the cerebral cortex in swallowing. Dysphagia 1993;8:195-202.

16. Smithard DG, O'Neill PA, Parks C, Morris J. Complications and outcome after acute stroke. Does dysphagia matter? Stroke 1996;27:1200-4.

17. Kidd D, Lawson J, Nesbitt R, MacMahon J. The natural history and clinical consequences of aspiration in acute stroke. QJM 1995;88:409-13.

18. Ertekin C. Voluntary versus spontaneous swallowing in man. Dysphagia 2011;26:183-92.

19. Teismann IK, Dziewas R, Steinstraeter O, Pantev C. Time-dependent hemispheric shift of the cortical control of volitional swallowing. Hum Brain Mapp 2009;30:92-100.

20. Watanabe Y, Abe S, Ishikawa T, Yamada Y, Yamane GY. Cortical regulation during the early stage of initiation of voluntary swallowing in humans. Dysphagia 2004;19:100-8.

21. Daniels SK, Brailey K, Foundas AL. Lingual discoordination and dysphagia following acute stroke: analyses of lesion localization. Dysphagia 1999;14:85-92.

22. Hamdy S, Aziz Q, Rothwell JC, Singh KD, Barlow J, Hughes DG, et al. The cortical topography of human swallowing musculature in health and disease. Nat Med 1996;2:1217-24.

23. Kwon M, Lee JH, Kim JS. Dysphagia in unilateral medullary infarction: lateral vs medial lesions. Neurology 2005;65:714-8. 
Sol Jang, et al.

24. Cola MG, Daniels SK, Corey DM, Lemen LC, Romero M, Foundas AL. Relevance of subcortical stroke in dysphagia. Stroke 2010;41:482-6.

25. Wan P, Chen X, Zhu L, Xu S, Huang L, Li X, et al. Dysphagia post subcortical and supratentorial stroke. J Stroke Cerebrovasc Dis 2016;25:74-82.

26. Heilman KM, Van Den Abell T. Right hemisphere dominance for attention: the mechanism underlying hemispheric asymmetries of inattention (neglect). Neurology 1980;30:327-30.

27. Howes D, Boller F. Simple reaction time: evidence for focal impairment from lesions of the right hemisphere. Brain 1975;98:317-32.

28. Smithard DG, O'Neill PA, Martin DF, England R. Aspiration following stroke: is it related to the side of the stroke? Clin Rehabil 1997;11:73-6. 